\title{
Comparison of CLIF-C ACLF Score and MELD Score in Predicting ICU Mortality in Patients with Acute-On-Chronic Liver Failure
}

Moazma Ramzan ${ }^{1}$, Ahtesham Iqbal ${ }^{1}$, Hafiz Ghulam Murtaza ${ }^{1}$, Nasir Javed ${ }^{2}$, Ghulam Rasheed ${ }^{2}$, Khadija Bano $^{1}$

1. Critical Care, Shifa International Hospital, Islamabad, PAK 2. Internal Medicine, Shifa International Hospital, Islamabad, PAK

Corresponding author: Moazma Ramzan, dr.moazzama@gmail.com

\section{Abstract}

\section{Introduction}

Acute-on-chronic liver failure (ACLF) is a serious complication of liver cirrhosis which presents with hepatic and/or extrahepatic organ failure and often needs admission to an Intensive Care Unit (ICU). This condition typically needs organ support and carries a high mortality rate. ICU care may not benefit these patients. There are many scores to assess prognosis in these patients, such as the Model for End-stage Liver Disease (MELD) score, the MELD score refined to take into account serum sodium level (MELD-Na), the chronic liver failure organ failure (CLIF-OF) score, the CLIF Consortium acute-on-chronic liver failure (CLIF-C ACLF) score and the Child-Turcotte-Pugh classification. This study was conducted to compare CLIF-C ACLF and MELD scores for selecting patients at risk of high mortality, as ICU care to these patients in the absence of liver transplantation may be of no value.

\section{Methods}

The data of 75 patients admitted to the ICU of Shifa International Hospital in Islamabad were prospectively analyzed. CLIF-C ACLF and MELD scores were calculated at admission and then at 24 and 48 hours after the ICU stay. Data were analyzed with the assistance of SPSS. Mortality was the primary outcome.

\section{Results}

Comparison of both scores showed that a CLIF-C ACLF score $\geqslant 70$ at 48 hours predicts mortality more accurately, with an area under receiver operating curve (AUROC) of 0.643 (confidence interval [CI] 95\% $0.505-0.781 ; \mathrm{p}=0.046$ ) which was significantly higher than MELD scores of 30,40 and 50 at 48 hours. Organ failure and the need for supportive care were strong predictors of mortality $(\mathrm{p}=<0.05)$.

Received 02/01/2020

Review began 02/18/2020 Review ended 02/20/2020 Published 02/24/2020

(c) Copyright 2020

Ramzan et al. This is an open access article distributed under the terms of the Creative Commons Attribution License CC-BY 4.0., which permits unrestricted use, distribution, and reproduction in any medium, provided the original author and source are credited.

\section{Conclusion}

We concluded that a CLIF-C ACLF score $\geqslant 70$ at 48 hours and organ failure are better predictors of mortality and that ICU care in these patients does not benefit them. Definitive therapy in the form of liver transplantation may have a promising role, if considered early.

Categories: Internal Medicine, Miscellaneous, Gastroenterology

Keywords: intensive care unit, mortality, clif-c aclf score, meld score

\section{Introduction}

Acute-on-chronic liver failure (ACLF) is a serious complication of hepatic cirrhosis, often requiring admission to ICU and organ support. Prognosis depends upon the number and severity of organ failures [1, 2]. Determining prognosis, therefore, helps to monitor treatment response, determine the need for emergency transplantation and provide the rationale for deciding on the futility or otherwise of ICU care.

ACLF is a very serious complication of hepatic cirrhosis that is characterized by hepatic and extrahepatic organ failure and is associated with high short-term mortality [3,4]. Such patients often require artificial support therapies and admission to an intensive care unit. Prognosis of ACLF is related to the number and severity of organ failures [1,2]. Thus, it becomes very important to determine prognosis, as prognosis can help to monitor response to treatment, determine the need for emergency transplantation and provide the rationale for deciding on the futility of ICU care.

The conventional scoring systems, the Model for End-stage Liver Disease (MELD) score, the MELD score refined to take into account serum sodium level (MELD-Na) and the Child-Pugh-Turcotte classification, were designed to predict the prognosis of chronic liver failure. These scoring systems are also commonly 
used to determine prognosis in ACLF. The accuracy of these scores in predicting the prognosis of ACLF is, however, limited by their inability to incorporate all possible extrahepatic organ failures, which are an important part of the disease spectrum and have a significant impact on prognosis.

Recently, new scoring systems, i.e., the chronic liver failure organ failure (CLIF-OF) score, the CLIF Sequential Organ Failure Assessment (CLIF-SOFA) score and the CLIF Consortium acute-on-chronic liver failure (CLIF-C ACLF) score have been developed and validated to predict short-term mortality in patients with ACLF $[5,6]$. Of these, the CLIF-C ACLF score has shown better accuracy in predicting mortality [5]. Since ACLF is a dynamic process, serial assessment of the score better predicts mortality and, therefore, can also help in assessing the futility or otherwise of organ support and ICU care

\section{Materials And Methods}

\section{Objective}

The objective of the study was to compare the CLIF-C CLF score and the MELD score in predicting ICU mortality.

\section{Study design}

This was a prospective cohort study.

\section{Sample size}

The total sample size was 75 , which was calculated by applying Buderer's formula for sample size calculation in sensitivity/specificity analysis, using the following parameters: Expected sensitivity (0.78) [5], Expected specificity (0.74) [5], Expected prevalence (0.88), Desired precision (0.10), and Confidence level (95\%).

\section{Diagnosis of ACLF and grading of severity}

ACLF was diagnosed using criteria derived from the CLIF OF classification, which is further derived from the CLIF-C SOFA score [3,4]. The CLIF-C ACLF was calculated using the following formula: CLIF-C ACLF $=10 \times$ $(0.33 \times$ CLIF- OFs $+0.04 \times$ Age $+0.63 \times \ln ($ WBC count $)-2)$ [3]. Organ failure was defined according to Moreau et al. [4]. Grade I ACLF was defined as having acute renal failure or any organ failure along with renal failure; Grade II as having two organ failures; Grade III and Grade IV as $\geqslant 3$ organ failures with a CLIF-C ACLF score $\leqslant$ 64 and 4 organ failures with a CLIF-C ACLF score $\leqslant 64$ respectively.

\section{Population and Study Settings}

The study was conducted at the Medical ICU/Medical Step Down facilities of the Shifa International Hospital in Islamabad for a period of six months. After approval from the institutional review board (IRB) and ethical committee, informed consent was taken from all participants. Patients of both genders aged > 18 years, admitted to the ICU/Medical Step Down facilities with a diagnosis of ACLF and associated organ failure were included in the study. Those patients whose families asked for withdrawal of intensive care and organ support, who transferred out of hospital $<48$ hours after admission to ICU and were $<18$ years of age were excluded from the study. Confidentiality was maintained throughout the study. A trainee researcher, who was blind of the objective of the study and adequately trained in filling proforma, recorded demographic and clinical data (serum bilirubin, serum creatinine, international normalized ratio (INR), oxygen pressure in arterial blood ( $\mathrm{PaO} 2)$, peripheral capillary oxygen saturation (SPO2), fraction of inspired oxygen (FiO2), requirement for mechanical ventilation or renal replacement therapy, use of vasopressors, and mean arterial blood pressure) on proforma within one hour of presentation at 24 and 48 hours after admission. The MELD score and the CLIF-C ACLF score were calculated using online calculators [7, 8]. The primary outcome was survival.

\section{Statistical methods:}

The collected data were entered and analyzed using the software, Statistical Package for Social Sciences, version 20.0 (SPSS). Descriptive data were expressed as percentages. Statistical analysis was performed to compare the two mortality groups of survivors and non-survivors. Multivariate logistic regression analysis was used to evaluate the risk factors. The receiver operating characteristics (ROC) curve was used to calculate the accuracy, sensitivity and specificity of the two scoring systems. A P-value of $<0.05$ was considered significant for all tests.

\section{Results}

We studied 75 patients. Of them, 37 (49.3\%) were males and 38 (50.7\%) were females. Most of the patients were aged between 51 and 60 years ( 37 patients, $49.3 \%$ ), and 31 patients were aged between 41 and 50 years (41.3\%) (table 1). Our results showed that there is high mortality (66.67\%) of ACLF patients with CLIF-C ACLF grade III or above, which is directly proportional to the grade of encephalopathy. Only 25 (33.3 \%) patients survived during their stay in ICU. Gender had no statistically significant effect on mortality. 


\section{Cureus}

Age

Age $<41$

41-50

$51-60$

$>61$
Percentage

$2 \%$

$41.3 \%$

$49.3 \%$

$7 \%$

TABLE 1: Age distribution of the study population

The areas under receiver operating curve (AUROC) for MELD scores 30, 40 and 50 at 48 hours were 0.532 (CI 95\% 0.388-0.675; $\mathrm{p}=0.658$ ), 0.594 (CI 95\% 0.455-0.734; $\mathrm{p}=0.190$ ) and 0.529 (CI 95\% 0.391-0.667; $\mathrm{p}=0.683$ ), respectively (figure 1 and table 2 ).

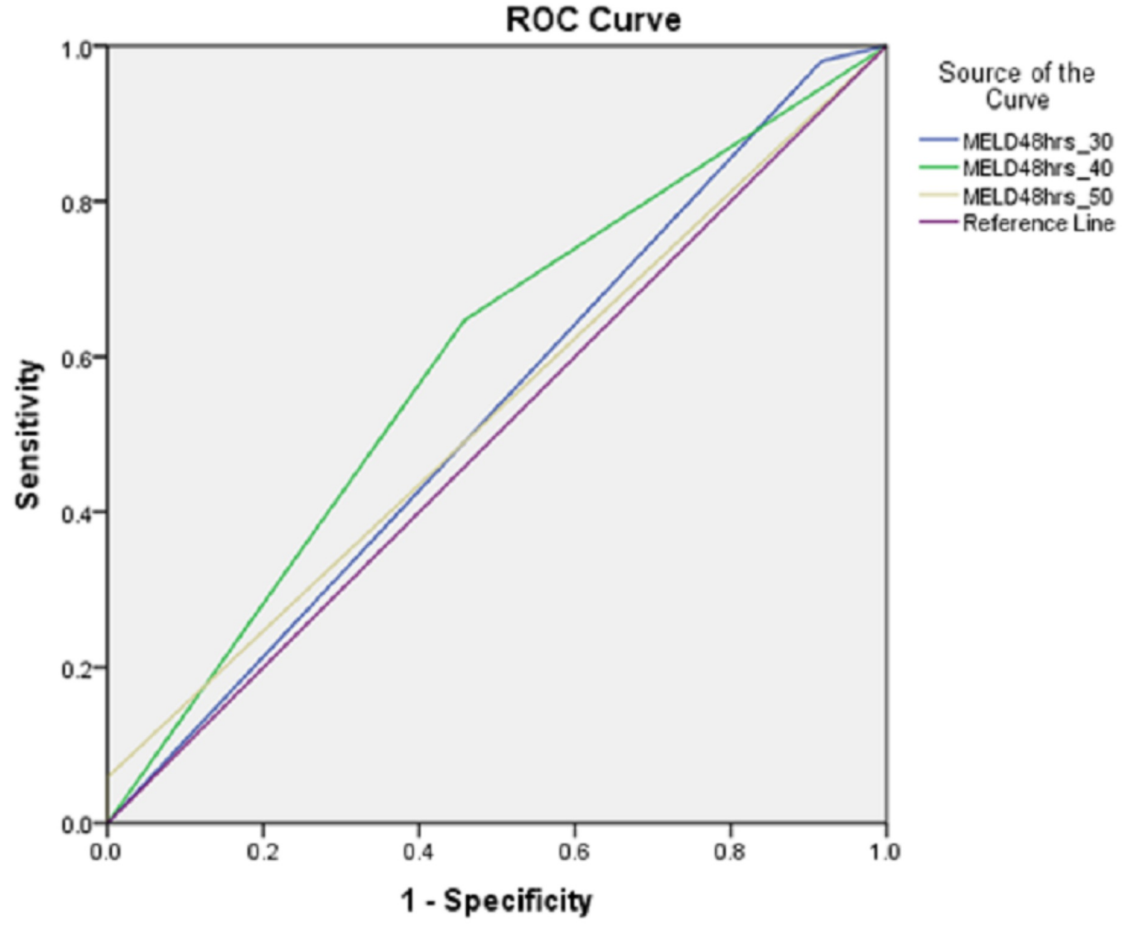

Diagonal segments are produced by ties.

FIGURE 1: Area under operator receiver characteristic for MELD score $30,40,50$ at 48 hours representing specificity 


\section{Cureus}

Area Under the Curve

\begin{tabular}{|c|c|c|c|c|c|}
\hline \multirow{2}{*}{ Test Result Variable(s) } & \multirow{2}{*}{ Area } & \multirow{2}{*}{ Std. Error ${ }^{\mathrm{a}}$} & \multirow{2}{*}{ Asymptotic Sig. ${ }^{b}$} & \multicolumn{2}{|c|}{ Asymptotic $95 \%$ Confidence Interval } \\
\hline & & & & Lower Bound & Upper Bound \\
\hline MELD48hrs_30 & .532 & .073 & .658 & .388 & .675 \\
\hline MELD48hrs_40 & .594 & .071 & . 190 & .455 & .734 \\
\hline MELD48hrs_50 & .529 & .070 & .683 & .391 & .667 \\
\hline
\end{tabular}

TABLE 2: The test result variable(s): MELD48hrs_30, MELD48hrs_40, MELD48hrs_50 has at least one tie between the positive actual state group and the negative actual state group. a. Under the nonparametric assumption. b. Null hypothesis: true area $=0.5$

The area under receiver operating curve (AUROC) values for a CLIF-C ACLF score $\geqslant 70$ at 0 hours, 24 hours and 48 hours were 0.498 (CI 95\% 0.356-0.639 p=0.973), 0.605 (CI 95\% 0.474-0.736; p=0.143) and 0.643 (CI $95 \% 0.505-0.781 ; \mathrm{p}=0.046$ ), respectively (figure 2 and table 3 ).

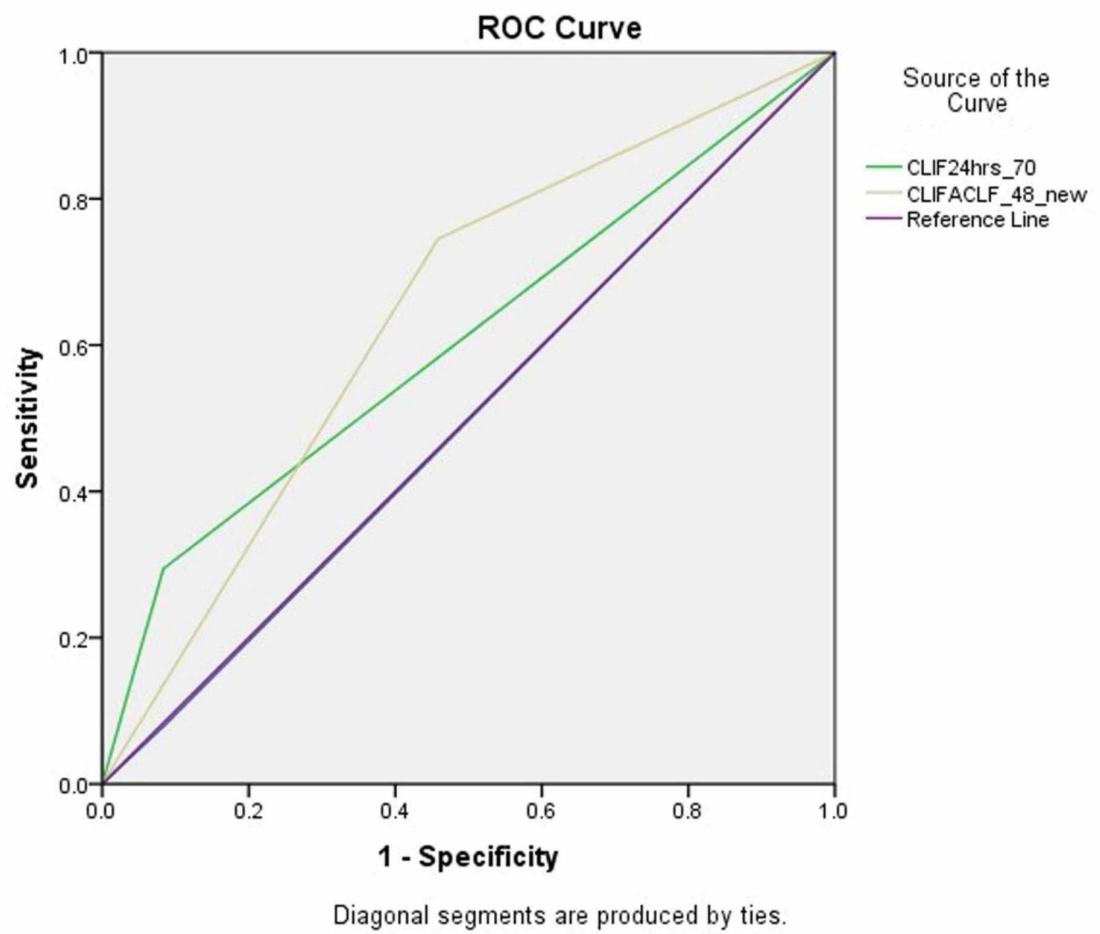

FIGURE 2: Area under receiver operating curve (AUROC) characteristics for CLIF-C-ACLF at 24 and 48 hours, representing specificity. The cutoff value for CLIF-C-ACLF was 70 


\section{Cureus}

\begin{tabular}{|c|c|c|c|c|c|}
\hline \multirow{2}{*}{ Test Result Variable(s) } & \multirow{2}{*}{ Area } & \multirow{2}{*}{ Std. Errora } & \multirow{2}{*}{ Asymptotic Sig. } & \multicolumn{2}{|c|}{ Asymptotic 95\% Confidence Interval } \\
\hline & & & & Lower Bound & Upper Bound \\
\hline CLFohrs 70 & 0.498 & 0.072 & 0.973 & 0.356 & 0.639 \\
\hline CLIF24hrs__70 & 0.605 & 0.067 & 0.143 & 0.474 & 0.736 \\
\hline CLIFACLF-48_new & 0.643 & 0.07 & 0.046 & 0.505 & 0.781 \\
\hline
\end{tabular}

TABLE 3: The test result variable(s): CLIFOhrs_70, CLIF24hrs_70, CLIFACLF_48_new has at least one tie between the positive actual state group and the negative actual state group. Symbol "a" denotes Under the nonparametric assumption whereas "b" stands for Null hypothesis: true area $=0.5$

The sensitivity and specificity of the CLIF-C ACLF score at 48 hours were $74.51 \%$ (60.37\% to $85.67 \%$ ) and $54.17 \%$ (32.82\% to $74.45 \%$ ) (CI $95 \%$ and $\mathrm{p}=<0.05$ ), respectively, whereas for MELD, scores of 30,40 and 50 at 48 hours had $98.04 \%$ (89.55\% to $99.95 \%$ ), $64.71 \%$ (50.07\% to $77.57 \%$ ) and $5.88 \%$ (1.23\% to $16.24 \%$ ) sensitivity (CI $95 \%$ and $\mathrm{p}=<0.05$ ), respectively. Specificity for MELD scores 30,40 and 50 at 48 hours was $8.33 \%$ ( $1.03 \%$ to $27.00 \%$ ), $54.17 \%$ ( $32.82 \%$ to $74.45 \%$ ), and $100 \%$ ( $85.75 \%$ to $100.00 \%$ ), respectively (CI $95 \%, \mathrm{p}=<0.05)($ table 4$)$.

\begin{tabular}{|c|c|c|c|c|}
\hline & Sesitivity & $(95 \% \mathrm{Cl})$ & Specificity & Specificity $(95 \% \mathrm{Cl})$ \\
\hline CLIF 48 & $74.51 \%$ & $60.37 \%$ to $85.67 \%$ & $54.17 \%$ & $32.82 \%$ to $74.45 \%$ \\
\hline MELD 30 & $98.04 \%$ & $89.55 \%$ to $99.95 \%$ & $8.33 \%$ & $1.03 \%$ to $27.00 \%$ \\
\hline MELD 40 & $64.71 \%$ & $50.07 \%$ to $77.57 \%$ & $54.17 \%$ & $32.82 \%$ to $74.45 \%$ \\
\hline MELD 50 & $5.88 \%$ & $1.23 \%$ to $16.24 \%$ & $100.00 \%$ & $85.75 \%$ to $100.00 \%$ \\
\hline
\end{tabular}

TABLE 4: Represents sensitivity and specificity of CLIF-C-ACLF at 48 hours and MELD scores of 30,40 and 50 at 48 hours.

The accuracy of the CLIF-C ACLF score and the MELD score in predicting survival was assessed by calculating the AUROC. A cutoff value was chosen to accurately predict fatalities with a high specificity. All potential confounders that were part of predictive score calculations for MELD and CLIF-C ACLF were not included in the multivariate model. Patients lost to follow-up were censored at the time of last patient contact. Normally distributed data are presented as mean $\pm \mathrm{SD}$ and nonparametric data are presented as median (IQR). A P value $<0.05$ was considered statistically significant.

\section{Predictors of Mortality}

Of the 75 patients, 50 did not survive, with 40 (80\%) patients requiring renal replacement therapy within 48 hours of ICU admission ( $\mathrm{P}=0.029$ ). Of the 25 survivors, 21 patients had grade II encephalopathy and four patients had grade III encephalopathy. Amongst the 50 non-survivors, 43 had grade IV encephalopathy (p= $<0.001$ ) and seven had grade III encephalopathy. Of the 50 non-survivors, 45 were on vasopressors at 48 hours of ICU stay $(p=<0.001)$. Of the 75 patients, 61 required invasive mechanical ventilation and, of those, 50 patients did not survive while 11 survived (table 5). 


\section{Cureus}

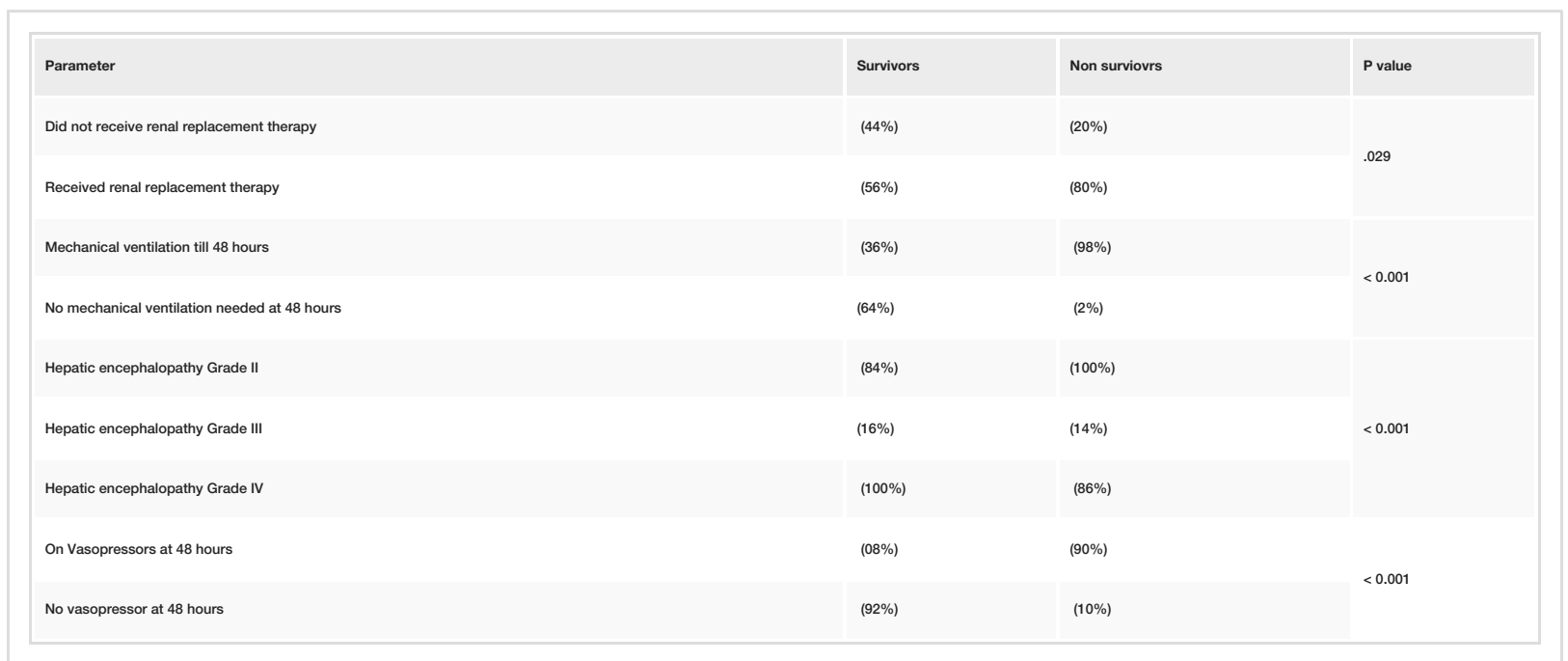

TABLE 5: Predictors of mortality

\section{Discussion}

Our data shows that ACLF carries a high mortality (\%), which is even higher in males compared to females, although this was not found to be statistically significant $(\mathrm{p}=0.259)$. A CLIF-C ACLF score $\geqslant 70$ at 48 hours is more accurate ( $74.51 \%$ sensitive and $54.17 \%$ specific) in predicting mortality in ICU than a MELD score at 48 hours. Although maximum treatment was offered to all patients, including optimizing the treatment of comorbidities and providing multidisciplinary care and full organ support, we found that as the MELD score increased, its sensitivity to predict mortality decreased, which challenges its utility in the ICU as a predictor of survival. Our findings are further supported by a study conducted in a tertiary care hospital in Portugal, which involved 289 patients and showed that the CLIF-C ACLF score is a better predictor of mortality than the MELD score (AUROC $0.799, \mathrm{p}<0.05$ ), with a reported specificity of $74 \%$ in comparison to our results (specificity $54.17 \%$, sensitivity $74.51 \%$ ) [9]. The difference between the two results may be due to our small sample size and the fact that we evaluated our results at 48 hours compared to 28 -day mortality, with the CLIF-C ACLF score of 70 as a cut-off compared to 50 in the other study.

Recently, in 2014, a multicenter study was published in the Journal of Hepatology which showed that a CLIF-C ACLF score $\leqslant 40$ has a $90 \%$ negative predictive value and \% sensitivity, whereas a CLIF-C ACLF score $\geqslant 60$ has an $82 \%$ positive predictive value and $94 \%$ specificity [6]. Although our sample size was small ( $\mathrm{n}=75)$, 46 patients had an ACLF-C ACLF score $\geqslant 70$ and mortality was (66.67\%), implying that patients with a high ACLF-C ACLF score have reduced liver reserves and limited regenerative ability. This may be the reason for the high mortality among these patients, despite receiving maximum treatment for precipitating factors and supportive care to failed organs. In order to reduce mortality in these patients, liver transplantation may be a way forward. The options of early liver transplantation and the risks, benefits and costs of the procedure should be discussed with the family at the time of the patient's presentation at ICU admission because the time between death and intervention may be very short. Otherwise, ICU care may be a futile effort to treat these patients. We did not follow the survivors (33.33\% of total participants) of our study after they were discharged from the critical care unit; hence, mortality beyond this cannot be predicted by our study. However, considering the high mortality within 48 hours of ICU admission, we anticipate that those who survived with high CLIF-C ACLF scores should be evaluated for early liver transplantation in an effort to improve their survival.

According to our above-mentioned results, organ failure and the number of organ failures are key for assessing the prognosis. We found that the need of vasopressors, mechanical ventilation, grade of hepatic encephalopathy and the need for renal replacement therapy were strong predictors of mortality in ICU, in spite of the delivery of best possible care. Liver transplantation in these settings may be a promising option for reducing mortality in these patients.

The MELD score is widely used to predict survival and, is a tool for deciding on candidacy for liver transplant patients. However, our data suggests that an increase in the MELD score is not a good predictor of mortality, raising concerns about whether it should be used among the criteria for liver transplantation. The CLIF-C ACLF score predicts mortality with more sensitivity and specificity at 48 hours, hence, it may be considered a better parameter than the MELD score for selecting patients for liver transplantation in ACLF.

\section{Conclusions}

We concluded that a CLIF-C ACLF score $\geqslant 70$ is a better predictor of ICU mortality in ACLF than the 
MELD score at 48 hours, and has higher sensitivity and specificity. Organ failures in ACLF are bad prognostic factors and, therefore, the provision of ICU care to these patients may be futile. ICU specialists should involve transplant team and families earlier to discuss the possibility of transplantation and cost of the procedure in order to provide definite treatment option to such patients. Further studies are needed to evaluate the effectiveness of CLIF-C ACLF scores for selecting patients of liver transplantation in ACLF.

\section{Additional Information}

\section{Disclosures}

Human subjects: Consent was obtained by all participants in this study. Institutional board review and ethical committee issued approval IRB\#163-653-2019. please note that with reference to your study entitled as " comparison of CLIF-C ACLF and MELD score in predicting ICU mortality" has been reviewed by IRB/EC. The IRB and Ethics Committee is pleased to approve the study. Animal subjects: All authors have confirmed that this study did not involve animal subjects or tissue. Conflicts of interest: In compliance with the ICMJE uniform disclosure form, all authors declare the following: Payment/services info: All authors have declared that no financial support was received from any organization for the submitted work. Financial relationships: All authors have declared that they have no financial relationships at present or within the previous three years with any organizations that might have an interest in the submitted work. Other relationships: All authors have declared that there are no other relationships or activities that could appear to have influenced the submitted work.

\section{References}

1. Bajaj JS, O’Leary JG, Reddy KR, et al.: Survival in infection-related acute-on-chronic liver failure is defined by extrahepatic organ failures. Hepatology. 2014, 60:250-256. 10.1002/hep.27077

2. Gustot T, Fernandez J, Garcia E, et al.: Clinical course of acute-on-chronic liver failure syndrome and effects on prognosis. Hepatology. 2015, 62:243-252. 10.1002/hep.27849

3. Jalan R, Yurdaydin C, Bajaj JS, et al.: Toward an improved definition of acute-on-chronic liver failure . Gastroenterol. 2014, 147:4-10.

4. Moreau R, Jalan R, Gines P, et al.: Acute-on-chronic liver failure is a distinct syndrome that develops in patients with acute decompensation of cirrhosis. Gastroenterology. 2013, 144:1426-37. 10.1053/i.gastro.2013.02.042

5. Engelmann C, Thomsen KL, Zakeri $\mathrm{N}$ et al.: Validation of CLIF-C ACLF score to define a threshold for futility of intensive care support for patients with acute-on-chronic liver failure. Crit Care. 2018, 22:254 10.1186/s13054-018-2156-0

6. Jalan R, Saliba F, Pavesi M, et al.: Development and validation of a prognostic score to predict mortality in patients with acute-on-chronic liver failure. J Hepatol. 2014, 61:1038-47. 10.1016/j.jhep.2014.06.012

7. CLIF-C ACLF (Acute-on-chronic liver failure) score and expected mortality rates . (2019). Accessed: February 24, 2020: http://www.efclif.com/scientific-activity/score-calculators/clif-c-aclf.

8. MELD score (original, pre-2016, model for end-stage liver disease) . Accessed: February 24, 2020 : https://www.mdcalc.com/meld-score-original-pre-2016-model-end-stage-liver-disease.

9. Barosa R, Ramos LR, Patita M, Nunes G, Fonseca J: CLIF-C ACLF score is a better mortality predictor than MELD, MELD-Na and CTP in patients with acute-on-chronic liver failure admitted to the ward. Rev Esp Enferm Dig. 2017, 109:399-405. 10.17235/reed.2017.4701/2016 